\title{
Biospheric approach as a compromise between hygienic and ecological approaches to radiological protection of the environment
}

\author{
R.M. Alexakhin ${ }^{1}$, S.V. Kazakov ${ }^{2}$ and I.I. Linge ${ }^{2}$ \\ ${ }^{1}$ The Agricultural Radioecology Research Institute, 249020 Obninsk, Russian Federation \\ ${ }^{2}$ Nuclear Safety Institute of the Russian Academy of Sciences (IBRAE RAS), \\ 52 B. Tulskaya St., 115191 Moscow, Russian Federation
}

\begin{abstract}
In the paper it demonstrates that for more precise and comprehensive interpretation under the framework of biospheric concept, the anthropocentric paradigm, being now the one of the basic principles in radiation protection, should be reformulated and amended (an immediate switch to ecological concept involves significant changes and calls for solution of very complicated scientific problems). It is suggested that application of the biospheric approach within the bounds of the modified hygienic principle allows resolving the majority of contradictions between anthropocentric and ecological approaches. This may represent a more perspective course of the radiation protection system development compared to a simple change of fundamental paradigms.
\end{abstract}

At present, the radiological protection of the environment is based upon ICRP Publication 60 provision which can be briefly summarized as follows: "Protected is man - protected are the biota" (hygienic or anthropocentric approach). Now there are both opinions for and against it.

Table 1. Arguments for and against the hygienic principle of radiological protection.

\begin{tabular}{|l|l|}
\hline For: & Against: \\
\hline $\begin{array}{l}\text { 1. Human is the most radiosensitive element of } \\
\text { biosphere. }\end{array}$ & $\begin{array}{l}\text { 1. There exist situations when human is absent } \\
\text { while man-caused radiation pollution influences } \\
\text { biota. }\end{array}$ \\
\hline $\begin{array}{l}\text { 2. Values of human permissible exposure are } \\
\text { considerably lower than those which cause } \\
\text { detectable negative human health effects. }\end{array}$ & 2. Human can protect himself from exposure. \\
\hline $\begin{array}{l}\text { 3. Protection of human health is of top priority as } \\
\text { a part of human activity. }\end{array}$ & $\begin{array}{l}\text { 3. Implicit form of biota radiation protection } \\
\text { ensuring. }\end{array}$ \\
\hline
\end{tabular}

Though this approach is not de jure fixed in the national radiation safety legislation (in federal laws or subordinate regulatory acts), it is de facto the basis of all practice that is carried out in this sphere. The modern Russian environmental protection legislation calls for ensuring radiation protection of the living organisms. This goes in line with the new international initiatives on development of principles and criteria for radiological protection of the biota (ecological approach).

The main difficulties of changing the radiation protection paradigm if the necessity of practical application of ecological principle of radiation protection will be theoretically and practically proved:

- Specification of exposure relative biological effectiveness and introduction the equivalent and effective doses for biota components.

- Referent plants and animals decision.

- Final plants and animals exposure effects which can be used as integral criteria of biota radiation protection.

- «Dose-response » relationship for referent plants and animals. 
- The role of biota radiation effects level (individual, population, ecosystem).

- Limits of biota exposure.

Comparison of the existing concepts for radiological protection (hygienic and ecological approaches) demonstrates that they are not mutually exclusive. With ecological approach, the ecosystem components are incorporated into the radiation protection system, and a "human" as a biosphere element may also be included into the system. From this standpoint, the biospheric approach may be considered as a more general one, combining both anthropocentric and ecological approaches. Such consideration makes the biospheric approach quite attractive from the scientific point of view and allows avoiding antagonism among different concepts. Moreover, with this interpretation the biospheric approach to provision of radiation safety reflects, to a large extent, modern scientific and philosophical viewpoints on society and science development processes.

Thus, in order to interpret hygienic approach in a more accurate and expanded way (in other words, to "transform" it to biospheric approach) in the context of using the approach in the radiation protection system as one of its basic principles, hygienic approach is needed to be reformulated and supplemented: - There is a need to introduce more clear and single valued term "critical group of population". At that, it is not necessary to treat it as a real social age group under the exposure but, possibly, as some virtual social age group which way of life leads to the maximum exposure.

- It is required to consider the worst (conservative) critical groups exposure situations (not necessary in real exposure situations).

- Exposure situations should include not only actual variants connected to the given point of time and the given place but also virtual exposure situations with the highest level of conservatism.

- Radionuclide migration in the environment and the process of dose formation in most attackable ecosystem components should be considered taking into account actual migration and accumulation variants for the specified ecosystem.

We should say that abovementioned additions mean radiation protection system to include not only principle of conservatism but also "protection of future generations" principle which is known to be one of the IAEA radioactive waste management principles. Not paying special attention to the important question of complete and consistent radiation protection system principles development, it should be stated that using both principle of conservatism and IAEA principles is fruitful and it helps obtaining informative results.

As a comparison measure between exposure of the human and ecosystem objects, it is possible to use index of radiation exposure (IRE). It is the ratio between actual received dose and its limit value (the limit value of dose for the human, according to the current legislation, is $1 \mathrm{mSv} / \mathrm{year}$; as to environmental objects, it is the maximum value of dose guaranteeing absence of any radiation-induced effects for the species).

As a variant of ecosystem where radionuclides are deposited to form human exposure, we consider simplified model conceptualization that 1 ) dose of radiation for the human is equal to the limit value of the dose, and 2) dose for the most attackable components of the model ecosystem reaches its maximum value.

Consider land ecosystem. If soil specific activity (for the given radionuclide) such that 1) human exposure reaches the maximum permissible level and 2) the level of soil organisms and soil biota exposure is so that $\mathrm{IRE}_{\text {biota }}<1$, then we may state that adherence to sanitary-hygienic standards of terrestrial environment pollution provides land ecosystems radiation protection.

In the table 2, dose assessments for land ecosystem components are given taking into account biospheric interpretation of hygienic approach. According to expectations, maximum exposure corresponds to more highly organized living matter. As to the values of $\mathrm{IRE}_{\text {biota }}$, even conservative assessments of this indicator are much less (approximately by two orders of magnitude) than 1, and only for monodelphs the value of IRE $\mathrm{Ebita}_{\mathrm{b}}$ is near 0.1 . It is very strong argument in favor of primacy of the hygienic approach to the environment radiation protection. 
Table 2. Dose assessment for land ecosystem components.

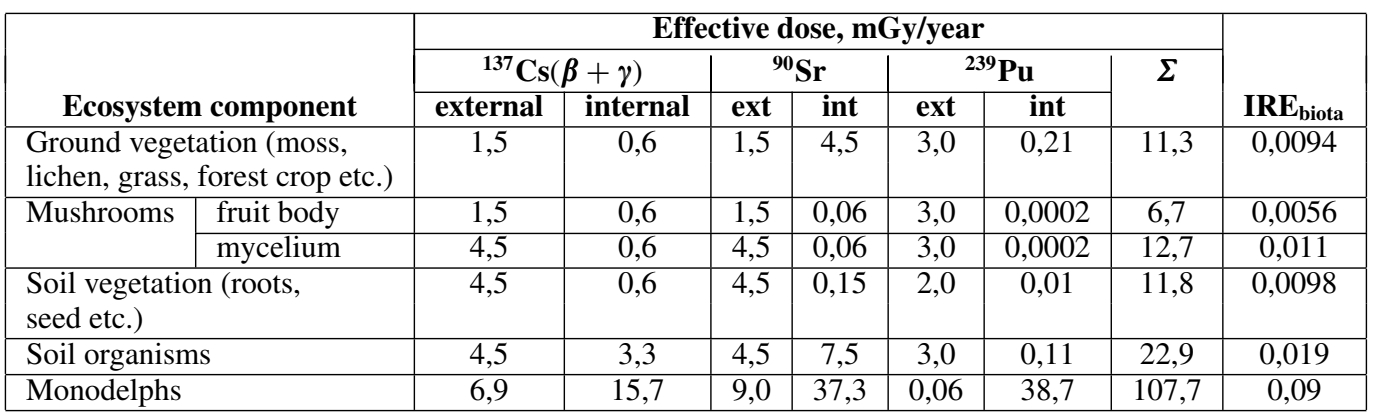

The essence of approach suggested is to supplement an "ecosystem-human" model with a certain "virtual human" who use resources of environment with no radiation restrictions (considering the present situation and future expansion of activity). We obtain a total conservatism: in terms of time, space and socially and age-specific status of human.

As to structure of radiation protection substantiation, taking into account advantages of both anthropocentric and Reference Animal and Plant approaches "virtual human" approach allows to reach higher level of abstraction. Essentially the method may be identified as "biospheric" where human loses its preferred position with respect to objects of environment in the view of mental and ethical principles. It is only considered in analysis that human is one of the most radiosensible elements of biosphere. It should be emphasized that "virtual human" radiation dose levels regulations guarantee radiation protection of non-human biota components.

The viewpoint reflects modern scientific and philosophical opinion on society, science and biosphere evolution. It also allows avoiding serious scientific and technological difficulties of changing the way of human and biota radiation protection ensuring. 
